Portland State University

PDXScholar

$11-22-2019$

\title{
How Urban Land Use Contributes to Inequitable Health Outcomes: an Interdisciplinary Review \& Analysis
}

Bliss Storm Croton

Portland State University

Follow this and additional works at: https://pdxscholar.library.pdx.edu/honorstheses

Part of the Environmental Public Health Commons

Let us know how access to this document benefits you.

Recommended Citation

Croton, Bliss Storm, "How Urban Land Use Contributes to Inequitable Health Outcomes: an Interdisciplinary Review \& Analysis" (2019). University Honors Theses. Paper 817.

https://doi.org/10.15760/honors.836

This Thesis is brought to you for free and open access. It has been accepted for inclusion in University Honors Theses by an authorized administrator of PDXScholar. Please contact us if we can make this document more accessible: pdxscholar@pdx.edu. 
How Urban Land Use Contributes to Inequitable Health Outcomes: An Interdisciplinary Review \& Analysis

How does neighborhood land use disparately impact population health; and what is the relationship between urban policy, socioeconomic status, and environmental discrimination within the context of psychological health outcomes?

Thesis

Bliss Croton

Portland State University 
Abstract

Using existing literature as a basis for initial review, this paper seeks to explore the relationship between urban land use and socioeconomic status as both pertain to the prevalence of psychological health diagnoses among neighborhood residents. The purpose of this analysis is to investigate the combined literature from multiple areas of research in order to gain a more holistic, up-to-date scope of knowledge regarding how discriminatory neighborhood land use may result in poorer psychological health outcomes for urban populations. By utilizing an interdisciplinary approach, some of the locally relevant, long-term impacts of uneven development and environmental injustice are examined based on how they synergistically contribute to psychological health outcomes. Frameworks of health ecology, urban political ecology, environmental justice, and social epidemiology provide a theoretical lens of assessment for impacts of various indicators on neighborhood health. The quality and quantity of accessible neighborhood green space is found to relate to differences in psychological well-being between neighborhoods of varying SES, with the prevalence of high-quality urban forests corresponding to a decline in negative outcomes and complaints regarding mental health. This suggests that urban greening may be an effective means of combating the growing problem of global psychological distress, as well as improving urban biodiversity. An essential guiding question of this review relates to how these frameworks may be applied in ways that support more equitable neighborhood health outcomes in cities like Portland, OR. 


\section{Foreword- A Note on Praxis}

It has been argued that it is not the consciousness of men that determines their being, but their social being that determines their consciousness (Marx, 1859). This assertion by Marx was made on the foundation that praxis provides a practical means of achieving revolutionary aims by enabling oppressed groups to informatively critique the system responsible for their subjugation. The validity of information is determined by the degree to which it supports the actualization of emerging progressive ideas and imparts meaningful action in recognition of such ideas. Understanding praxis as the process through which a theory becomes realized is essential to addressing the questions I am asking in this paper, and thus also relevant to my own perspective and experiences as a community-oriented aspiring researcher. My interest in the subjects that comprise this literature review initially grew out of my desire to understand the hidden workings of my environment, and more specifically, make sense of why the world was built in ways that provided some with so little and others with so much, based on what seemed to be arbitrary factors. This curiosity was intensified by the moving I did with my family throughout my childhood, from extremely rural areas to small towns to the suburbs, all prior to settling in Portland. It was clear to me even then that our sense of place has a huge impact on the way a person feels about themselves and their ability to prioritize health, this truth would be underscored by my later role as a member of an undergraduate research team tasked with unearthing and publicizing the racial covenants found in the housing deeds of formerly redlined Portland neighborhoods. This experience marked an appreciation for the idea that neutral space does not exist, but rather unequal power exchanges define the built environment. Such a reality is especially true in cities, both because the environment is predominantly socio-naturally constructed and because of the ways in which urban environments perpetuate the elitist interests of the sociopolitical groups who built them in the first 
place. Portland is a fascinating case study of the perceptual disconnect between the new waves of gentrification and old cycles of disinvestment, the dichotomy of which has also guided my interest in environmental and spatial injustice. An interdisciplinary investigation of the literature will aid in raising awareness of these intersecting issues and increasing the involvement of new voices in the problem-solving discourse, as well as supporting informed advocacy. By intertwining my own experiential knowledge with empirical research findings and critical theories such as ecology, I hope to contribute to a more nuanced space in which community researchers are able to contribute the value of their own unique perspectives to the work they do.

\section{Introduction}

This paper seeks to investigate aspects of the relationship between land use and indicators of neighborhood socioeconomic status as both relate to psychological health. Recent work states that the current total global burden of mental illness is underestimated by more than a third, indicating a need for further research into the underlying causes of mental health (Vigo, Thornicroft, \& Atun, 2016). The purpose of this approach is to point out how our current state of the literature needs to incorporate a greater understanding of the ways in which land use, when wielded as a means of environmental discrimination, can lead to worse psychological health outcomes for certain populations. Incorporating this interdisciplinary perspective allows factors such as uneven development to be assessed according to the degree to which they contribute to psychological health outcomes. Additionally, this perspective may be valuable (or simply interesting) when applied to local housing, health, and/or neighborhood development policies in Portland and other cities. The amount and type of green space present in residential areas is significantly associated with reducing negative 
health outcomes such as cardiovascular disease, premature birth rates, depression, and all-cause mortality (Rojas-Rueda, Nieuwenhuijsen, Gascon, Perez-Leon, \& Mudu, 2019). This delving of public health, psychology, urban environmental policy, and ecology literature is intended to assess the socioeconomic factors inherent in the built environment of urban spaces with a focus on the placement, quantity, and quality of neighborhood green spaces in relation to psychological health. This interdisciplinary approach is intended to illustrate the current state of the relationship between the historically inequitable construction of urban space and the various health disparities faced by neighborhood populations that may have been disproportionately affected by this power dynamic both in the past and up to the present (Krieger, 2001). For the purposes of this research question, this review will focus particularly on any connection between neighborhood socioeconomic status indicators and issues that negatively affect mental health, such as depression and anxiety. Mental health is defined by the World Health Organization as "a state of well-being in which [an] individual realizes [their] own potential, can cope with the normal stresses of life, can work productively and fruitfully, and is able to make a contribution to [their] community” (Bratman, Anderson, Berman, Cochran, de Vries, Flanders, \& Daily, 2019). Examining these dynamics within the context of the intentional disinvestment and deprioritization that often plagues minority group neighborhoods will allow for increased dialogue concerning the long-term health implications of these actions on future generations (Petteway, Mujahid, Allen, \& Morello-Frosch, 2019). The inclusion of these factors will aid in the aim of this research to explore how the uneven development and use of urban space may impact the variability of mental health outcomes among neighborhood residents. This is necessary because mental health is a rising global concern (Vigo, 2016). 
The two issues of spatial justice and health equity are intersectional in how they reflect the influence that controllable social dynamics and disparities -such as the organization and politics of physical space- have on health outcomes, however this relationship is still being inadequately addressed within modern public health literature and warrants continued discussion. There is also a need for a greater scope of acknowledgement of the roots and reasons for many of these ongoing disparities, which can reinvigorate cycles of generational trauma and contribute to poorer psychological outcomes. How do intersecting factors such as urban land use and SES simultaneously perpetuate disparate psychological health outcomes for residents in neighborhoods that have experienced patterns of environmental discrimination? This investigation will examine differing narratives of health and explore how synthesizing various theoretical perspectives may lay out the groundwork for potential answers to this broad inquiry.

\section{Methods}

This research was conducted primarily in the form of a literature review of articles pertaining to the intersection of urban land use, perceived neighborhood satisfaction, environmental injustice, and psychological health outcomes. A search for peer-reviewed literature within this sphere was conducted using PubMed cross-referenced with MEDLINE, Web of Science, ProQuest, PsycNET, ScienceDirect, and Google Scholar. Articles within these areas were selected based on the criteria of having a recent publication date, being conducted in the form of quantitative research, and including indicators of both health and land use that were relevant to this research question. For example, many articles that were not selected matched other criterion for the search but focused exclusively on physiological health outcomes rather than or to the detriment of discussing psychological health 
outcomes. A recent publication date was defined as 2010 onward for quantitative, empirical sources, whereas more leeway was afforded to theoretical and historically contextual sources such as Gibson (2007) and Krieger (2001 and 2012). This was done because many early theoretical arguments in favor of incorporating mental health into public and environmental health analysis gained traction in the 1990's through the first decade of the 2000's; however most viable experimental research on the topic has been done within the past 10 years. Additionally, studies conducted prior to 2010 were deemed for the most part to be outdated or limited in their methods, and thus ill-suited to the purpose of this current literature review. Search terms used for these databases included health outcomes, health equity, environmental discrimination OR injustice, social determinints of health, neighborhood socioeconomic status indicators, psychological health (e.g. mental health; depression; anxiety) urban political ecology, classic ecology, health ecology, socio natural, population health (e.g. neighborhood health; urban health; health AND urban policy) land use (e.g. green equity; urban forest; spatial discrimination) uneven development, and social epidemiology. This approach allowed for a more comprehensive overview of how discussions surrounding land use, psychological health, and injustice serve to frame the question of interest, as well as greater insight into how interdisciplinary literature approaches both the causes and the improvement of population health disparities. This context enabled a better focus for review on the quality and quantity of urban green space in regards to health and how neighborhood indicators of health are perceived on an individual level.

\section{Narratives in the Literature}




\section{Background}

This section addresses the literatures that were identified as satisfying the search criteria and contributing to a meaningful discourse. The discussion is organized into subsections so as to best illuminate the interdisciplinary coverage of the overarching research question. These subsections include an introductory background, a brief discussion of psychological health outcomes, an outline of concepts pertaining to land use, and a reflection based on ecological frameworks of analysis.

There is a distinct lack of equity among urban neighborhoods when it comes to land use, particularly related to both the quantity and quality of green space such as urban forest and foliage. Considering differentiations such as size and type of green space are important, as these characteristics mediate mental health in unique and various ways (Akpinar, Barbosa-Leiker, \& Brooks, 2016). One indicator of low neighborhood SES is a clearly defined lack of access to high-quality green spaces, as well as a general decrease in the average quantity of urban tree canopy and vegetation. Neighborhood SES is relevant to determining variability in health outcomes due to the differing quality of the green space that is typically made available to different groups, as higher SES groups have been found to enjoy greater access to high-quality green spaces than lower SES groups (Rojas-Rueda et al, 2019). Additionally, high SES neighborhoods are less likely to be impacted by some of the negative aspects of green space, such as the ways increased desirability and cost-of-living can further contribute to gentrification. Some indicators of high-quality green space are perceived quality, size of area, degree of accessibility, volume of visitations, surrounding greenness, foliage diversity, and walkability (Leslie \& Cerin, 2008). In many neighborhoods, the quality of the accessible green space is compromised by neglect, environmental contaminants, and/or a scarcity of enrichment in the space itself. As an extension of environmental discrimination, this is closely tied to the historic disenfranchisement and 
de-prioritization of the communities that reside in these neighborhoods which has also contributed to a pattern of less optimal health outcomes for members of these communities (Krieger, 2012). The basic idea of environmental discrimination evolved from that of environmental racism, which refers to the way in which minority group neighborhoods are both burdened with a disproportionate number of physical environmental hazards and receive inadequate regulatory protection from those hazards in comparison to non-minority groups due to racially-biased policies (Abel \& White, 2011). A primary example of unevenly dispersed exposure can be observed in the air quality across many cities across the U.S., including Seattle, where unequal patterns of industrial toxic air have converged in minority group areas that have experienced sociohistoric discrimination (Abel \& White, 2011). In many contemporary studies of environmental injustice, minority group neighborhoods are those that are populated primarily by people of color and/or members of low socioeconomic groups. The concept of environmental discrimination as a unique, concrete form of injustice originated from research done in environmental epidemiology, which is the study of aspects of the physical environment and their implications for the distribution of human disease (Krieger, 2012). According to McLaren \& Hawe's (2004) definition of environmental epidemiology, this research has traditionally focused on factors contributing to the contamination of air, water, and food; and has recently broadened to also consider the disproportionate impacts of global climate change. Although much of this past work has been concerned with the relationship between the environment and physically adverse health outcomes such as reproductive health and cancers, there is also evidence linking environmental factors to psychological outcomes such as anxiety, depression, and even a rise in suicidal ideation worldwide (McLaren \& Hawe, 2004). 
A lack of adequate urban green space is correspondingly linked to a greater proportion of industrial land use, increased environmental pollution, and a decrease in community-focused investment. All of these factors have been shown to negatively impact health by lowering birth weights, causing higher rates of respiratory illnesses and increases in chronic conditions such as cancer (Cusack, Larkin, Carozza, \& Hystad, 2017). Conversely, high-quality neighborhood green spaces provide health benefits such as increased immune and metabolic function, reduced cardiovascular illnesses, improved maternal and natal outcomes, and better psychological well-being (Rojas-Rueda et al, 2019). Exposure to high-quality urban foliage has been shown to improve residential health outcomes in regards to mental, physical, and social wellness, whereas no exposure or exposure to low-quality greenery is correlated with lower neighborhood satisfaction. Part of the reason that measures of satisfaction matter in relation to health is that several main indicators of neighborhood satisfaction (such as safety, noise, and spatial characteristics) have also been shown to act as independent predictors of mental health (Leslie \& Cerin, 2008). This implies that neighborhood satisfaction may mediate certain aspects of the association between perceived physical characteristics of space and measures of mental wellness in adults. This demonstrates how spatial equity matters in a physical sense, as uneven development or use of neighborhood land leads to disparate perceptions of different spaces. The notion of spatial equity can describe the equitability present in any given socioeconomic exchange that occurs in a space, such as those involving goods and services. It is therefore important to understand spatial equity as both a process and an outcome resulting from the process. Due to their innately sensitive connection to the socionaturally produced environment, urban forests can be anticipated to be affected by these localized processes, making them an interesting and multi-faceted proxy measure for neighborhood health. Socio natural environments are generally 
described as those in which humans interact with nature, specifically describing those processes which intersect to create a uniquely constructed environment such as a city (Bentley, 2014). The conception of socio natural as a term led to some of the earliest attempts to define urban dynamics and, together with metabolic flow, contributed to the development of classic urban ecology as a theory (Heynen, Kaika., \& Swyngedouw, 2006). As such, this concept is central to the overarching framework of ecology and highly relevant to population health.

\section{Health Outcomes}

There is currently much more research exploring how environmental quality contributes to physical health outcomes like these than there is in relation to mental health outcomes such as depression and anxiety, though there is growing evidence that suggests the extent to which our mental wellbeing is intrinsically tied to our individually-perceived environmental context (Gianaros \& Manuck, 2010). The study of psychological health outcomes for historically marginalized communities, and minority groups in particular, warrants greater attention and resources (Krieger, 2001). Due to a growing recognition of the impact that mental illness can have on overall health and wellbeing, both individual and community level factors should be taken into consideration when assessing population risk. This is necessary given that the fraction of the total global burden of disease that is deemed attributable to mental illness has recently been estimated to be as high as $32 \%$ of total years lived with disability (YLD) and 13\% of disability-adjusted life-years (DALYs) (Bratman et al, 2019). These rates are comparable to those of cardiovascular and circulatory diseases, yet psychological conditions such as mood and anxiety disorders have been frequently neglected or treated as an afterthought in past population studies of environmental health (Bratman et al, 2019). Having the 
access and opportunity to engage with nature settings in a meaningful way has been shown to positively impact sleep and reduce chronic stress, both of which have a meaningful impact on an individual's risk of experiencing mental illness. Exposure to varying types of green space, a modifiable environmental factor, functions as one upstream method for promoting community health, lowering psychological distress, and reducing mortality (Astell-Burt \& Feng, 2019). One reason that different types of green space may contribute to variance in mental health outcomes is that high-SES neighborhoods may enjoy more diverse, aesthetically-pleasing, well-curated green space than low-SES neighborhoods, which results in an unbalanced sense of perceived satisfaction between residents of the two areas (Leslie \& Cerin, 2008). Of relevance here is the fact that the majority of urban trees are on private residential land, and as such their health and abundance are both dependant on the class currently occupying said land. Research shows that the type of green space one has access to matters in terms of vegitative complexity, meaning that it is more beneficial for overall health to be exposed to dense foliage such as a tree canopy than to sparser foliage such as a grassy, open space (Astell-Burt \& Feng, 2019). In this article, while any amount of green space corresponds with lower odds of psychological distress, exposure to tree canopy in particular was associated with lower psychological distress and higher self-ratings of general health. Tree canopies are known to be supportive of higher levels of biodiversity (one key example being birds), which previous studies suggest are associated with more favorable psychological well-being. These findings imply that increasing the quantity of tree canopy through urban greening strategies may be a dualistically effective means of addressing community mental health concerns and ecological degradation. This research runs the risk of being confounded by a range of socioeconomic and demographic factors like income, employment, and education; as well as other factors such as age, sex, and relationship status. These personal 
socioeconomic circumstances pertain to varying forms of social privilege, and are each independently associated with disparate mental health risks in ways that must be adjusted for when assessing population health outcomes (Reiss, 2013). Additionally, more research is needed in order to determine whether the potential benefits of exposure to high-quality green spaces are long-term in their causal role.

\section{Land Use}

Land use has historically meant the level of maintenance and alteration inflicted by humans onto their natural environment; and also involves the creation and management of our built environment (Sharma, Gardner, \& Begbie, 2019). Urban land use describes this relationship specifically as it occurs in cities, a dynamic often uniquely marked by changes to the landscape, demographic transitions, and the economic, social, and environmental impacts introduced by urbanization. Urbanization involves the outward expansion of population centers beyond their original limits, and has become an increasingly urgent topic of conversation among public health professionals due to the large number of people gravitating towards city centers around the world. It is projected that over $65 \%$ of the global population will live in urban areas by 2050 , meaning it is critical to understand the costs, benefits, and disparate impacts of urbanization as they relate to vulnerable populations (Sharma et al, 2019). One example of a potential health risk arising from urban environments is their association with a decrease in residential access to nature (Bratman et al, 2019). Urban metabolism, also referred to as metabolic flow, expands the idea that cities behave in ways similar to a living organism, and is used as a means to model complex systems of exchange such as those described by spatial equity (Heynen et al, 2006). This concept incorporates a contemporary 
understanding of how class and privilege are innately woven into the infrastructure of our surroundings. By nature, the systems of exchange constructed within our socio natural environments are inequitable, making such environments an issue of equity and justice (Bentley, 2014). Recognition of this fact is crucial to any discussion of the built environment. Thus, metabolization can be further viewed as a function of abstract socioeconomic processes related to urban restructuring (Heynen et al, 2006). The process of urbanization as we know it in the United States is borne through a rapid version of metabolization as dictated by economic incentives, which can include cycles of environmental destruction and revitalization. These cycles of disinvestment have been and continue to be deeply rooted in racism, typically involving the intentional framing of community interests as being in opposition to each other as a way to obscure or justify discriminatory motives (Gibson, 2007). A commonly known example of this is "urban decay", which refers to the sociological and structural decline of a previously functional urban area. This decline is the result of systematic, institutional neglect by the financially responsible parties (such as political figures responsible for dispersing public funds), which often do not represent individuals or the interests of the population actually living in the affected locality (Gibson, 2007).

Such neglect is a reflection of the ways in which the needs of minority groups are frequently deprioritized by disempowering and undermining the consumption funds of low-income neighborhoods. Consumption funds are also known as cycling of resources, and describes the development, distribution, and use of the income that is used for the social accumulation of nonproductive capital within a community. It includes skills or knowledge, goods and commodities, and settings or events (McLaren \& Hawe, 2004). A consumption fund can also describe the quantity of financial resources available to a community for a particular purpose, such as maintaining healthy 
urban forests. If the collective neighborhood income is low, this reduces their consumption fund, which in turn increases their local dependency on the distal forces in charge of determining the distribution of general funds. As stated, these forces are generally unrepresentative of the communities for which they allocate resources, so this dynamic ensures that the power to selectively distribute funds is retained outside the primarily impacted population. An additional outcome of this asymmetric relationship is the tendency to then hold vulnerable populations responsible for the upkeep of their land, despite withholding access to adequate resources. Since Portland was founded, the city has been no exception to this inequitable process of urbanization. It is worth noting that prior research has been done to document the racially discriminatory history of many Portland neighborhoods, most notably in north and northeast Portland (Gibson, 2007). This existing work, along with the broader discourse within social epidemiology and health ecology, has contributed significantly to the contextual framework for my research.

\section{Ecological Frameworks}

This research is largely built upon various iterations of the ecological model as a means of highlighting the relevance of socioeconomic indicators in relation to health exposures and, subsequently, disparate health outcomes (Bentley, 2014). This relationship is known to have negative effects on the wellbeing of individuals who, for financial, historical, or sociopolitical reasons, are more likely to be exposed to environmental toxins such as concentrated industrial emissions on a chronic, long-term basis (Abel \& White, 2011). They are also less likely to be able to benefit from positive environmental exposure, resulting from either access to poor-quality space or a lack of access altogether. Additionally, these community members face more barriers when it comes to accessing care 
due to lack of insurance, financial means, or simply transportation to a treatment facility. The health promotion ecological perspective is a conceptual framework designed to equally weigh both individual and environmental determinants of behavior, which reflects the reality underlying most health decisions more accurately than a moral or behaviorist framework (McLaren \& Hawe, 2004). Central to this particular ecological perspective is the interaction and reciprocal causation between levels of influence. When applying this framework to public health, features include understanding health as a pattern of relations rather than as a single quantitative outcome as well as dialogue between the natural and social sciences that allows room for the explanation of how physical and social processes equally contribute to health (McLaren \& Hawe, 2004). Another theory of ecology that is important in terms of environmental and population health is that of urban ecology, which for our purposes is split into two distinct branches: classic urban ecology and urban political ecology. Classic urban ecology uses physical characteristics to define 'environment' and explain the relationship between ecological and social processes of cities as being one of metabolic flow (Heynen et al, 2006). This model has been criticized by some scholars, including Heynen, as being biologically reductionist in nature, which has thus contributed to the rising popularity of the alternate model of urban political ecology (UPE). This theory emerged more recently as a product of the theoretical and empirical synthesization of many previous urban environmental approaches, including environmental justice, classic ecology, and marxism. It further develops classic urban ecology's notion of metabolism with the purpose of unravelling the perceived neutrality of change so as to understand the power-relations inherent to the production of inner-city environments. This is especially relevant because these dynamics are directly tied to patterns of intentional investment and disinvestment, as well as the production of labor power within these communities. The main points to take away from UPE are situated within this 
interpretation of research encompassing the relations of power present in built environments, and recognize the degree to which the material environment is structured to benefit the elite above all others. Acknowledgement of this fact is necessary for concluding that there are few or no spaces that are unaffected by human processes of consumption and change, and that this is especially true of cities in the 21 st century. Because of the underlying economic, political and cultural processes inherent to urban landscape production, such change tends to be spatially differentiated and highly uneven in its rate of development. Persistent differences in levels of socioeconomic development between and among different sectors of society speaks to the idea that the restructuring of urban spaces is inherently political, and inextricably tied to issues such as deindustrialization and gentrification of non-productive land (Smith, 1984.) As a result of this disparity, overpopulation and poverty cannot be held responsible as the cause of environmental degradation and resource depletion. Rather, these ills serve to reflect the human cost of such degradation and depletion. UPE and theories related to it are relevant to the question of interest because of the ways in which urban socionatural metabolization operates as a function of broader socioeconomic processes such as land use and community investment. An understanding of how past urban land use policies have impacted contemporary neighborhood dynamics is necessary in order to constructively analyze existing mental health disparities between urban populations.

Essential to these assertions is the concept of embodiment, which examines the way in which humans internalize facets of our environment into our bodies and requires a discussion of how discrimination hurts health in a multitude of ways (Krieger, 2012). This concept seeks to incorporate socionatural causes into our contemporary understanding of the aetiology of disease, and utilizes social epidemiological theories such as the political economy of health as one method of analysis (Krieger, 
2001). Social epidemiology as a discipline is concerned with the socio-environmental factors associated with patterns of disease in populations. Within this framework, social experiences such as social support/isolation, social status and status (in)con-sistency, work conditions, society and social organization are considered to be direct influences on health. This makes it similar in approach to social ecology, which pays explicit regard to the social, institutional, and cultural contexts relating to people and their environment. Ecological theories that incorporate epidemiologic perspectives such as this are useful because they allow for a more nuanced view of environments and health, particularly when viewing socioeconomic status from a generational perspective. Doing exactly this is essential in reconsidering how certain communities experience green space and nature, as enriching naturalistic environments become progressively more and more unavailable to marginalized urban neighborhoods. This propagates a harmful feedback loop of younger urban generations becoming increasingly disconnected from the green space present in their communities, in addition to being psychologically disinherited from their collective right to the same full spectrum of nature experiences as claimed by individuals from more privileged neighborhoods. Finally, this results in a fading standard of what constitutes a healthy, viable green space or urban forest in comparison to a norm of increased environmental pollution. All of these factors contribute to a phenomenon that could be referred to as disembodiment, where a shifting point of reference spirals into a generational obliteration of environmental memory and ownership.

\section{Discussion}

In short and in all obviousness, humanity and nature are not separate entities. Rather, the two coexist within a socio naturally constructed urban environment that allows individuals to see 
themselves as distinct from their surroundings and exempt from the processes that alter said surroundings. Many of these processes may have been initially inorganic but have become self-sustaining in perpetuation, such as the self-fulfilling cycle of neighborhoods affected by urban decay. This situation is a result of deliberate human action done with the purpose of suppressing specific communities, and continues to have a wide array of effects on individuals living in marginalized areas. Changes in land use are directly correlated to the control of capital and affected by human processes of metabolization and development. Urban forests and tree canopy has been observed to fluctuate in relation to other urban consumption patterns such as construction, while also simultaneously being indicative of the ways that socioeconomic variables such as resource access, transportation options, and degree of financial investment can contribute to neighborhood SES and in turn to health outcomes. Finding oneself in or nearby high-quality green space may assist in building capacities for improved mental health, contribute to the recovery of impaired or compromised cognitive capacities, aid in recuperation from acute psychosocial stress, and even increase feelings of optimism (Astell-Burt \& Feng, 2019). The evolution of critical social and environmental justice research plays an important role in raising awareness of this truth, which can be observed using various ecology frameworks to deconstruct the power relations present within urban consumption patterns. By evaluating urban development through the combined perspectives of neighborhood land use and environmental health equity, this serves to highlight the disparities that have been born from chronic disinvestment and institutionalized urban neglect. In addition to operating as proxy measures for a degree of societal oppression and relegation, these disparities continue to manifest through embodiment in ways that impact the health of communities, and they will worsen further without a deeper understanding of the roots of this issue. 


\section{Conclusion}

Recognition of the power structures that dictate patterns of consumption is necessary in order to understand the layers of exploitation that have been codified into the development of urban space by way of zoning, resource allocation, and cultural visibility. Although the scope of this review and analysis was limited in its ability to delve deeply into key causal factors and identities, it attempts to expand explorations of local injustice through a broader lens of place and policy in connection to health. Indications for future research include further exploring the ways in which urban environmental interventions like canopy restoration, heat island mitigation, and improved biodiversity may help mediate negative mental health outcomes. They also may include the continued development of quantitative place-health measurements, increased advocacy for the health needs of marginalized neighborhoods, and enhanced efforts to expose points of intervention along individual pathways of embodiment. With that said, it is time to reexamine the process of urban environmental change as being linked to the construction and inevitable consumption of the urban built environment. When integrated into existing geographic ideas as a way of illustrating how various socioeconomic processes impact urban environments we can also better understand how these processes alter quality of life in an urban space. Further, such a perspective can be used to assess the effectiveness of urban revitalization projects at improving psychological health. Tools such as this better equip us to approach the following question: is change within the built environment neutral or is it transactional in terms of prioritizing the health of some communities over the health of others? 


\section{References}

Abel, T., \& White, J. (2011). Skewed Riskscapes and Gentrified Inequities: Environmental Exposure Disparities in Seattle, Washington. The American Journal of Public Health, 101. https://doi.org/doi:10.2105/AJPH.2011.300174

Akpinar, A., Barbosa-Leiker, C., \& Brooks, K. R. (2016). Does green space matter? Exploring relationships between green space type and health indicators. Urban Forestry Ev Urban Greening, 20, 407-418. https://doi.org/10.1016/j.ufug.2016.10.013

Astell-Burt, T., \& Feng, X. (2019). Association of urban green space with mental health and general health among adults in australia. JAMA Network Open, 2(7), e198209.

https://doi.org/10.1001/jamanetworkopen.2019.8209

Bentley, M. (2014). An ecological public health approach to understanding the relationships between sustainable urban environments, public health and social equity. Health Promotion International, 29(3), 528-537. https://doi.org/10.1093/heapro/dat028

Bratman, G. N., Anderson, C. B., Berman, M. G., Cochran, B., de Vries, S., Flanders, J., ... Daily, G. C. (2019). Nature and mental health: An ecosystem service perspective. Science Advances, 5(7), eaax0903. https://doi.org/10.1126/sciadv.aax0903 
Cusack, L., Larkin, A., Carozza, S. E., \& Hystad, P. (2017). Associations between multiple green space measures and birth weight across two US cities. Health E̊ Place, 47, 36-43.

https://doi.org/10.1016/j.healthplace.2017.07.002

Gianaros, P. J., \& Manuck, S. B. (2010). Neurobiological pathways linking socioeconomic position and health. Psychosomatic Medicine, 72(5), 450-461.

https://doi.org/10.1097/PSY.0b013e3181e1a23c

Gibson, K. (2007). Bleeding Albina: a history of community disinvestment, 1940-2000. Transforming Anthropology, 15(1), 3-25.

Heynen, N., Kaika, M., \& Swyngedouw, E. (2006). In the nature of cities - Urban political ecology and the politics of urban metabolism. ResearchGate.

Krieger, N. (2001). Theories for social epidemiology in the 21st century: an ecosocial perspective. International Journal of Epidemiology, 30(4), 668-677. https://doi.org/10.1093/ije/30.4.668

Krieger, N. (2012). Methods for the Scientific Study of Discrimination and Health: An Ecosocial Approach. American Journal of Public Health, 102(5).

https://doi.org/doi:10.2105/AJPH.2011.300544 
Leslie, E., \& Cerin, E. (2008). Are perceptions of the local environment related to neighbourhood satisfaction and mental health in adults? Preventive Medicine, 47(3), 273-278.

https://doi.org/10.1016/j.ypmed.2008.01.014

Marx, K. (1859). A Contribution to the Critique of Political Economy. Retrieved from https://www.marxists.org/archive/marx/works/1859/critique-pol-economy/preface.htm

McLaren, L., \& Hawe, P. (2004). Ecological perspectives in health research | GLOSSARY.Journal of Epidemiology and Community Health, 2005(59), 6-14. https://doi.org/10.1136/jech.2003.018044

Petteway, R. J., Mujahid, M., Allen, A., \& Morello-Frosch, R. (2019). The body language of place: A new method for mapping intergenerational "geographies of embodiment" in place-health research. Social Science $\sigma^{2}$ Medicine, 223, 51-63. https://doi.org/10.1016/j.socscimed.2019.01.027

Reiss, F., (2013). "Socioeconomic inequalities and mental health problems in children and adolescents: A systematic review," Social Science $6^{\circ}$ Medicine, Elsevier, vol. 90(C), pages 24-31.

Rojas-Rueda, D., Nieuwenhuijsen, M. J., Gascon, M., Perez-Leon, D., \& Mudu, P. (2019). Green spaces and mortality: A systematic review and meta-analysis of cohort studies. The Lancet Planetary Health, 3(11), e469-e477. https://doi.org/10.1016/S2542-5196(19)30215-3 
Sharma, A., Gardner, T., \& Begbie, D. (2019). Approaches to water sensitive urban design. Elsevier. https://doi.org/10.1016/C2016-0-03594-5

Smith, N. (1984). Uneven development: Nature, capital, and the production of space. New York, NY: Blackwell.

Vigo, D., Thornicroft, G., \& Atun, R. (2016). Estimating the true global burden of mental illness. The Lancet Psychiatry, 3(2), 171-178. https://doi.org/10.1016/S2215-0366(15)00505-2 